\begin{tabular}{|c|l|}
\hline Title & Plasmonic Enhancement of Raman Scattering on Non-SERS-A ctive Platinum Substrates \\
\hline Author(s) & Ikeda, Katsuy oshi; Sato, Jun; Fujimoto, Norihiro; Hay azawa, Norihiko; Kawata, Satoshi; Uosaki, Kohei \\
\hline Citation & $\begin{array}{l}\text { Journal of Physical Chemistry C, 113(27), 11816-11821 } \\
\text { https://doi.org/10.1021/p901858t }\end{array}$ \\
\hline Issue Date & 2009-07-09 \\
\hline Doc URL & http://hdl.handle.net/2115/50212 \\
\hline Type & article \\
\hline File Information & JPCC113-27_11816-11821.pdf \\
\hline
\end{tabular}

Instructions for use 


\title{
Plasmonic Enhancement of Raman Scattering on Non-SERS-Active Platinum Substrates ${ }^{\dagger}$
}

\author{
Katsuyoshi Ikeda, ${ }^{*, \hbar,}$ Jun Sato, ${ }^{\ddagger}$ Norihiro Fujimoto,${ }^{\ddagger}$ Norihiko Hayazawa, ${ }^{\S}$ Satoshi Kawata, ${ }^{\S, \|}$ \\ and Kohei Uosaki*, ${ }^{*}$ \\ Physical Chemistry Laboratory, Division of Chemistry, Graduate School of Science, Hokkaido University, \\ Sapporo 060-0810, Japan, RIKEN, Wako, Saitama, 351-0198, Japan, Department of Applied Physics, \\ Osaka University, Suita, Osaka 565-0871, Japan, and International Center for Materials Nanoarchitechtonics \\ Satellite (MANA), National Institute for Materials Science (NIMS), Sapporo 060-0810, Japan
}

Received: February 28, 2009; Revised Manuscript Received: April 19, 2009

\begin{abstract}
Gap-mode plasmon excitations on non-SERS-active metal substrates are investigated theoretically and experimentally for Pt single crystalline surfaces. A huge enhancement with the order of $10^{5}$, which is $10^{3}$ fold larger than the previously reported values, is experimentally achieved on Pt substrates. Since the present technique is applicable for a well-defined smooth substrate, crystal-face dependence of adsorption geometries is also presented at $\operatorname{Pt}(100)$ and $\operatorname{Pt}(111)$. These features indicate that the concept of gap-mode plasmon excitations is practically useful for studying catalytic reaction mechanism on various metal surfaces.
\end{abstract}

\section{Introduction}

Surface enhanced Raman scattering (SERS) is widely recognized as a powerful spectroscopic tool for studying molecular adsorption geometries on metal surfaces. ${ }^{1,2}$ After the discovery of the SERS effect $^{3-5}$ significant efforts have been made to increase the enhancement factor. As a result, the factor is now approaching the order of $10^{14}$, implying a possibility of single molecular detection. ${ }^{6,7}$ However, the practical use of SERS is limited to roughened surfaces of coinage metals such as Ag, $\mathrm{Au}$, and $\mathrm{Cu},{ }^{8}$ and unfortunately, most of other metals, including catalytic metals such as Pt and Pd, are commonly considered to be non-SERS-active. ${ }^{9,10}$ Since information of metal-molecular interactions is of essential importance to understand catalytic reaction mechanisms, many researchers have tried to maximize SERS effect on Pt-group metals. So far, the obtained enhancement factor on Pt is limited to the order of $10^{2}$ because of highly damping optical responses of these metals. ${ }^{9,10}$ A thin layer of non-SERS-active metals formed on a SERS-active substrate is also utilized for studying molecular species on catalytic surfaces. ${ }^{11,12}$ However, such a thin metal layer could have a different electronic property from that of the bulk metal. ${ }^{13-15}$

Enhancement of Raman scattering intensity is closely related to excitation of surface plasmon polaritons on a metal surface. ${ }^{1,2}$ Therefore, even when a SERS-active metal is used as a substrate, the appearance of SERS effect is strongly dependent on surface morphology of the substrate. On a smooth surface, for example, no SERS effect is present because the conservations of energy and momentum between photons and surface plasmons are not satisfied. In order to make photon-plasmon coupling allowed, electrochemically roughened substrates are usually utilized in conventional SERS measurements. ${ }^{3-5}$ However, since various adsorption sites are exposed on such substrates, information of each adsorption site is not available and SERS spectra provide

\footnotetext{
'Part of the "Hiroshi Masuhara Festschrift".

* Corresponding authors. E-mail: kikeda@ pchem.sci.hokudai.ac.jp (K.I.); uosaki@pcl.sci.hokudai.ac.jp (K.U.).

* Hokkaido University.

$\S$ RIKEN.

"Osaka University.

${ }^{\perp}$ NIMS.
}

only averaged information. This is also a serious limitation of SERS spectroscopy.

Recently, we have reported a convenient technique for efficient enhancement of Raman signal intensities on atomically flat Au-single crystalline substrates. ${ }^{16}$ This technique is based on excitation of localized gap-mode plasmons, ${ }^{17-21}$ in contrast to conventional SERS, which gains intensity from propagating surface plasmons. While surface plasmon propagation is largely affected by the magnitude in the imaginary part of a dielectric constant, the localized plasmon resonance property is only due to the real part. ${ }^{22}$ Hence, one can expect this gap-mode technique is effective even for non-SERS-active metals.

In this paper, we report gap-mode enhanced Raman scattering of organic monolayers on non-SERS-active Pt substrates. Platinum is recognized as one of the most important catalytic metals. Indeed, Pt-catalysts are now indispensable for operation of fuel cells and purification of automotive exhaust gas. Therefore, it is very important to know how molecules interact with a specific crystal face or a defect-site of platinum. Well resolved gap-mode enhanced Raman spectra were obtained for self-assembled monolayers (SAMs) of 4-chlorophenyl-isocyanide (CPI) on atomically flat $\mathrm{Pt}(111)$ and (100) single crystalline faces. Enhancement factor at Pt single crystalline surfaces is found to be comparable to that at Au single crystalline surfaces. Remarkable crystal-face dependence is presented in adsorption structures of the SAMs.

\section{Theory}

Since a well-defined metal-molecular interface is our concern, a so-called sphere-plane system, ${ }^{17,18}$ consisting of a metal nanopaticle and a smooth metal substrate, is used as a model resonance system for gap-mode plasmon excitation (see Figure 1a). When the metal sphere is far from the metal plane, electromagnetic interactions are negligible; incoming optical photons can excite only lowest-order dipole plasmons localized on the sphere. As the sphere approaches the plane, however, collective oscillation modes of electrons are excited in the system via electromagnetic interactions between the sphere and the plane. Importantly, these collective plasmon modes, so-called gap-modes, are accompanied with highly localized electromag- 
(a)

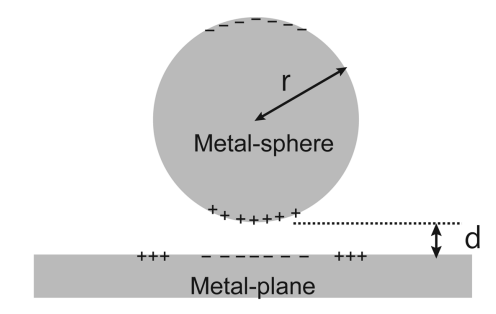

(b)

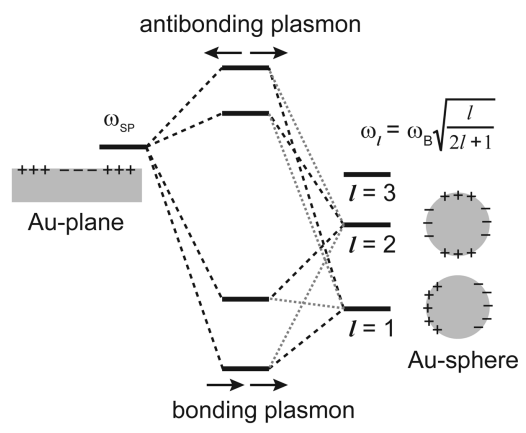

(c)

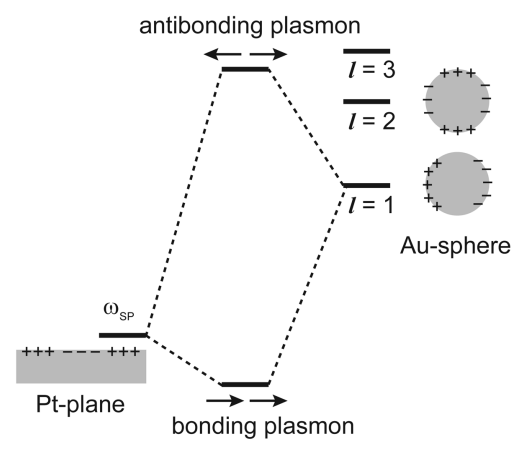

Figure 1. (a) Schematic illustration of a sphere-plane system for gapmode plasmon excitation, and plasmon hybridization diagrams for (b) $\mathrm{Au}$-sphere/Au-plane system and (c) Au-sphere/Pt-plane system.

netic field in the small spatial region between the sphere and the plane, with the volume of $\sim \mathrm{nm}^{3}$. Therefore, huge enhancement of Raman scattering intensity is expected in the gap region even on a flat surface.

Such an interaction in the resonance system can be understood by the plasmon hybridization theory. ${ }^{23,24}$ The degree of hybridization is dependent not only on the gap distance but also on the combination of metal species. For example, the energy level of surface plasmons on the Pt plane is lower than that on the $\mathrm{Au}$ plane by $2.8 \mathrm{eV}$ because of the difference in the electron density. As a result, the hybridization of the Au sphere with the Pt plane is expected to be different from that with the Au plane. Figure 1b,c shows schematic illustrations of expected plasmon hybridizations in Au sphere/Au plane and Au sphere/ Pt plane systems, respectively. It is considered that higher-order multipole plasmon contributions $(l=2,3, \ldots)$ from the $\mathrm{Au}$ sphere is relatively weak in the case of the Pt plane.

Figure 2 shows theoretically calculated extinction spectra of the Au sphere/Au plane and Au sphere/Pt plane systems. The calculation is carried out for $p$-polarized illumination with the incident angle of $45^{\circ}$, by taking into account the multipole interactions under a static field approximation, according to Wind's method. ${ }^{22,25}$ In the case of the $\mathrm{Au}$ sphere/Au plane (Figure 2a), the peak position is significantly red-shifted and the peak intensity is increased with decreasing the gap distance. As shown in Figure 1b, the red-shift of plasmon resonances is a characteristic feature of the bonding plasmons hybridized with multipole plasmons. As already explained, such a multipole
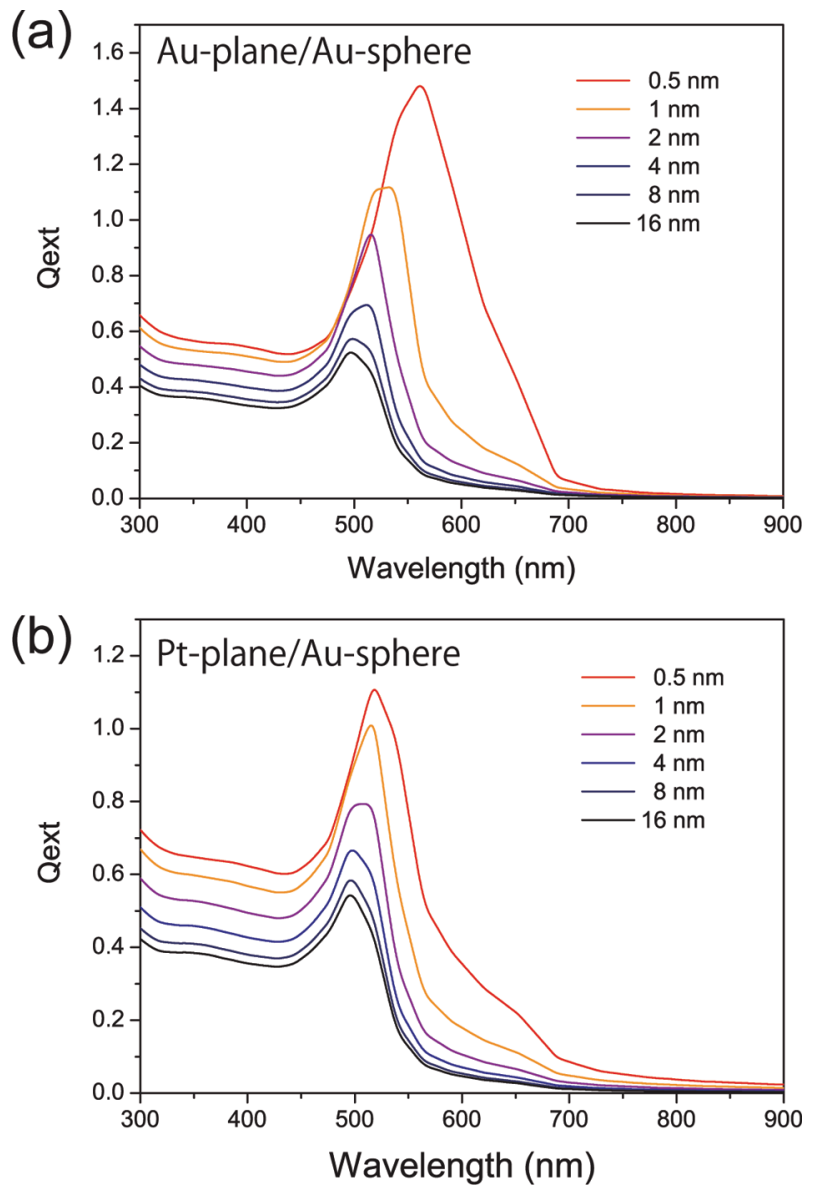

Figure 2. Calculated extinction spectra of (a) Au sphere/Au plane system and (b) Au sphere/Pt plane system with various gap-distances. The Au-sphere diameter was fixed to $20 \mathrm{~nm}$. Both systems are illuminated by $p$-polarized light with an incident angle of $45^{\circ}$.

plasmon contribution would be less in the Au-sphere/Pt-plane system. As shown in Figure 2b, the peak shift is indeed smaller in this system. Even so, the plasmon resonances are still found to be in the wavelength region of 600-700 nm when the gap distance is less than $1 \mathrm{~nm}$. Thus, one can expect that Raman scattering intensity is enhanced even on a Pt plane when the excitation wavelength is selected to be within this region.

This possibility was examined by finite difference time domain (FDTD) calculations simulating electric field distribution of an $\mathrm{Au}$ sphere/Pt plane, as well as that of an $\mathrm{Au}$ sphere/Au plane, excited by $p$-polarized 632.8-nm $\mathrm{He}-\mathrm{Ne}$ laser radiation. As shown in Figure 3, both systems clearly showed localization of electric field in the gap regions. Although the enhanced electric field at the Pt plane is weaker than that at the Au plane as expected, the local field enhancement is more than 20 with the gap distance of $1.5 \mathrm{~nm} .{ }^{26}$ When Raman shifts are small, the enhancement of Raman scattering is given by the fourth power of the field enhancement. ${ }^{27}$ That should be large enough for detection of scattering signals from organic monolayers.

\section{Experimental Section}

The sphere-plane system for gap-mode plasmon excitation was experimentally constructed as follows. ${ }^{16} \mathrm{~A} \mathrm{Pt}$ - or Au-single crystalline microbead, having atomically flat (111) and (100) facets, was prepared by the Clavilier method. ${ }^{28}$ As for the Ptcrystal, additional annealing was carried out at $1600 \mathrm{C}^{\circ}$ for $6 \mathrm{~h}$ under argon/hydrogen flow by using an induction heater (HOTSHOT-2 kW, Ameritherm), and then the surface was 

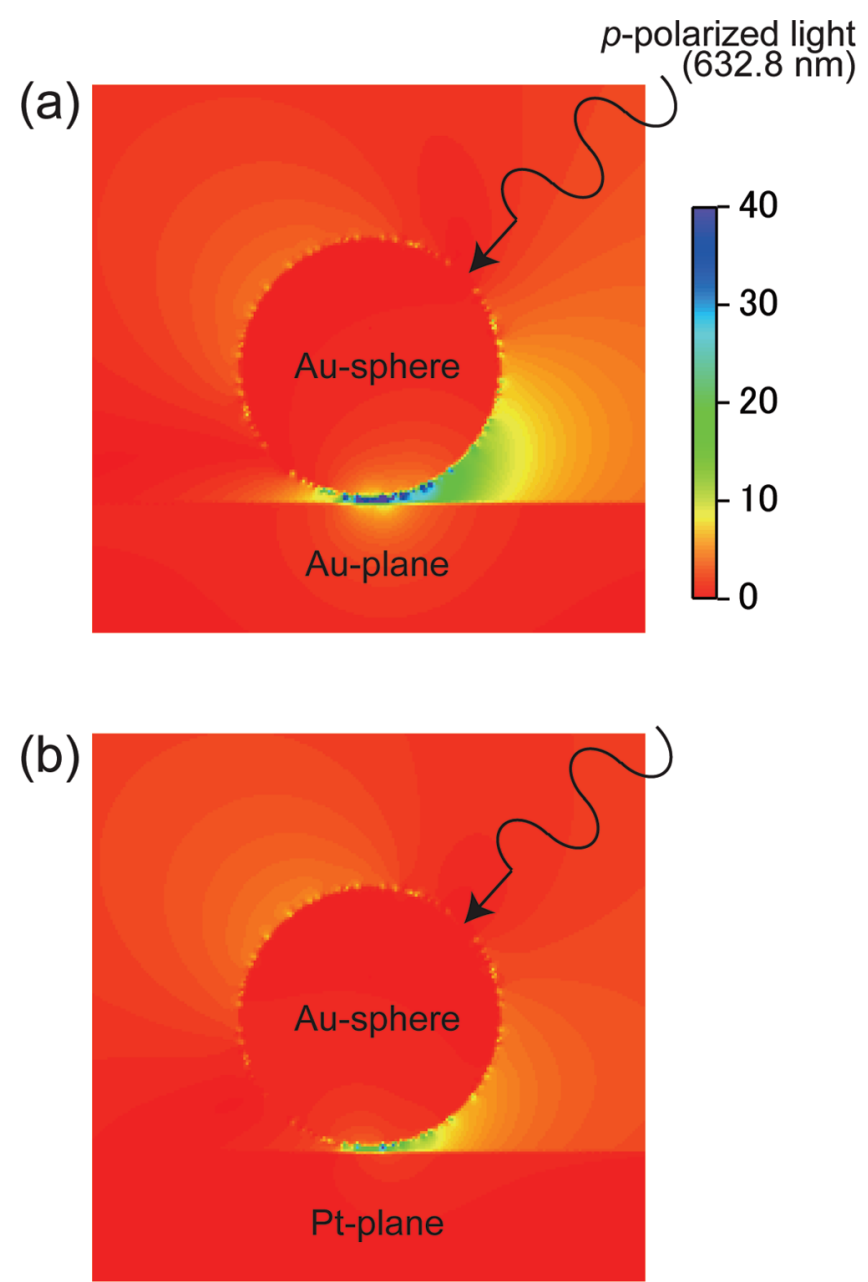

Figure 3. Electric field distribution calculated by FDTD for (a) Au sphere/Au plane and (b) Au sphere/Pt plane systems under a $p$-polarized illumination at $632.8 \mathrm{~nm}$ with an incident angle of $45^{\circ}$. The gap distance and the diameter of the $\mathrm{Au}$ sphere were set to be 1.5 and $50 \mathrm{~nm}$, respectively. ${ }^{25}$

protected by a hydrogen-saturated pure water droplet. ${ }^{29} \mathrm{CPI}$ was chosen as a Raman scatterer because the NC stretching vibration of CPI is sensitive to metal substrates. SAMs of this molecule were formed on $\mathrm{Pt}$ and Au single crystal surfaces from a THF solution containing $10 \mathrm{mM}$ CPI, which was obtained from Oakwood products and used as received, under argon atmosphere. Finally, the metal substrates covered with SAMs were dipped in a colloidal solution of Au nanoparticles (Au NPs) with diameter of ca. $20 \mathrm{~nm}$, prepared by the citrate reduction method $^{30}$ so that the Au NPs were adsorbed on the organic layers. Figure 4 shows SEM images of the CPI-SAM/Au(111) and the CPI-SAM/Pt(111) surfaces after the immersion in the colloidal solution of Au NPs. Although it is generally considered that $\mathrm{Au}$ NPs do not adsorb on alkyl SAMs without anchoring head groups, these SEM images confirm that Au NPs can be adsorbed on aromatic SAMs. The driving force could be the dispersion force but the detailed adsorption mechanism is still under investigation. Au NPs were well dispersed and typical coverage of $\mathrm{Au}$ NPs was around $20 \%$ for both of Pt and $\mathrm{Au}$ substrates.

A microscope system was used to selectively observe Raman scattering signals from each facet. Details of the measurement system were reported elsewhere. ${ }^{16}$ Briefly, the sample was illuminated through an objective lens $(40 \times, 0.6$ N.A.) by $632.8-$ $\mathrm{nm}$ radiation with intensity of $0.02 \mathrm{~mW}$ from a $\mathrm{He}-\mathrm{Ne}$ laser, (a)

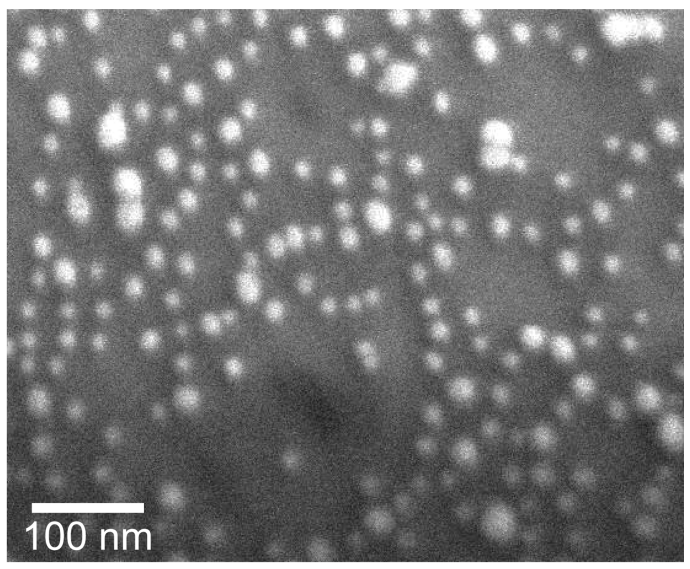

(b)

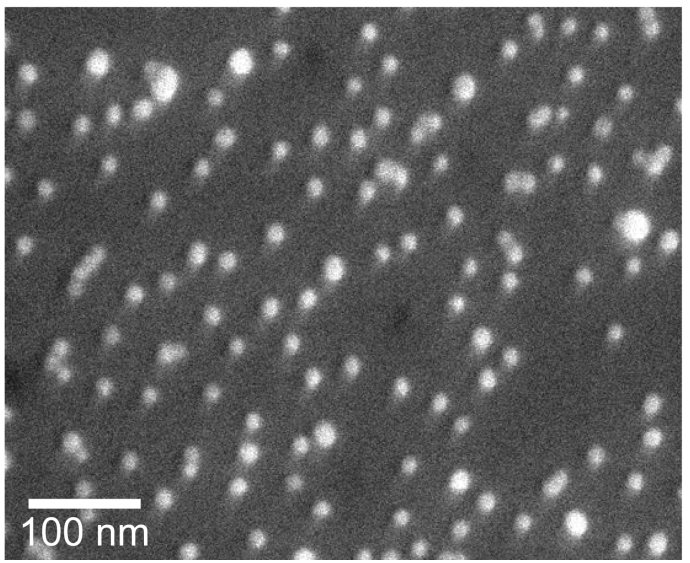

Figure 4. SEM images of (a) Au NPs/CPI-SAM/Au(111) and (b) Au NPs/CPI-SAM/Pt(111).

and backscattered Raman signals were collected with the same objective lens and monitored by a CCD-polychromator system (PIXIS 400B, Princeton Instruments) after Rayleigh scattering light was filtered by an edge filter (LP02-633-RU, Semrock). Note that the tight focusing with the high N.A. object lens is of essential importance to excite gap-mode plasmons and collect scattered Raman signals from the gap regions.

\section{Results and Discussion}

Figure 5a shows typical Raman spectra of a CPI monolayer on a $\mathrm{Pt}(100)$ surface. Without adsorption of Au NPs (top panel), no Raman signal was observed as expected because surface plasmon polaritons were not excited on the smooth Pt surface. By adsorption of Au NPs (bottom panel), the signal intensity was substantially enhanced, and five distinct vibrational modes with $\mathrm{A}_{1}$ symmetry were clearly observed at $1089(v \mathrm{C}-\mathrm{Cl}, 13)$, $1168(\sigma \mathrm{CH}, 9 \mathrm{a}), 1208(\nu \mathrm{C}-\mathrm{NC}, 7 \mathrm{a}), 1586(\nu \mathrm{C}=\mathrm{C}, 8 \mathrm{a})$, and 2145 $\mathrm{cm}^{-1}(\nu \mathrm{NC}) .{ }^{31,32}$ Among these modes, vibrational frequencies of $v \mathrm{C}-\mathrm{NC}$ and $\nu \mathrm{NC}$ are highly sensitive to metal substrates. The $v$ NC peak was observed at $2145 \mathrm{~cm}^{-1}$ at the $\mathrm{Pt}(100)$ surface (Figure 5a), whereas it appeared at $2185 \mathrm{~cm}^{-1}$ at the $\mathrm{Au}(100)$ surface (Figure $5 b$ ). Such a peak shift is reasonably explained by considering the difference in the degree of $\pi$ back-donation; the LUMO of CPI is a $\pi^{*}$ orbital that is antibonding with respect to the $\mathrm{NC}$ bond and any electron donation into this orbital from metal $\mathrm{d}_{\pi}$ orbitals leads to a decrease in the $v \mathrm{NC}$ frequency. ${ }^{33}$ These results clearly show that the information of CPI/Pt interfaces can be indeed obtained by this method. ${ }^{34}$

We now focus on the estimation of the enhancement factor of the gap-mode system at Pt substrates. As shown in Figure 5, 
(a) $\operatorname{Pt}(100)$

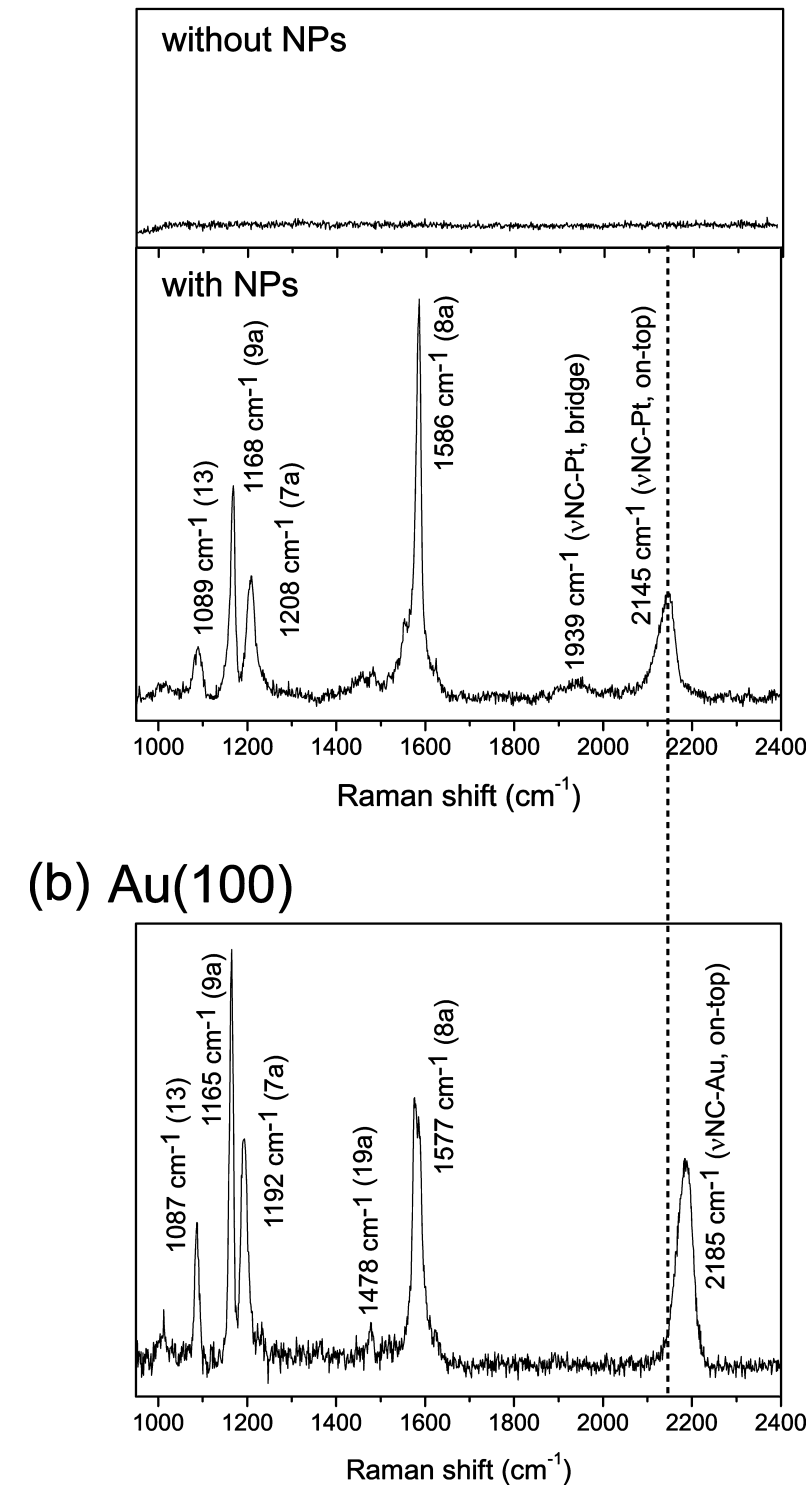

Figure 5. (a) Raman spectra of CPI-SAM at the Pt(100) facet without (top panel) and with (bottom panel) Au NP adsorption. (b) Raman spectrum of CPI-SAM at $\mathrm{Au}(100)$ with $\mathrm{Au}$ NP adsorption. These measurements were carried out by $632.8-\mathrm{nm}$ excitation through the objective lens with N.A. of 0.6.

the adsorption geometries of CPI on $\mathrm{Pt}(100)$ and $\mathrm{Au}(100)$ are similar as the positions of $v \mathrm{NC}$ peaks indicate that the on-top configuration is dominant in both cases. Although the small peak at $1939 \mathrm{~cm}^{-1}$ at $\mathrm{Pt}(100)$ surface suggests the presence of small fraction of the bridge configuration, ${ }^{31,32}$ it is negligible for the rough estimation. Besides, the molecular packing density was nearly equal at both surfaces as measured by ellipsometry. Therefore, the relative enhancement factor at the Pt substrate to the enhancement factor at the Au substrate should be roughly equal to relative intensity of the Raman peak at the $\operatorname{Pt}(100)$ surface to the Raman peak intensity at the $\mathrm{Au}(100)$ surface. Since the areas of Raman peaks at $\operatorname{Pt}(100)$ surface relative to those at $\mathrm{Au}(100)$ surface are $0.39(\nu \mathrm{C}-\mathrm{Cl}), 0.32(\sigma \mathrm{CH}), 0.36$ $(\nu \mathrm{C}-\mathrm{NC}), 0.38(\nu \mathrm{C}=\mathrm{C}),{ }^{35}$ and $0.31(\nu \mathrm{NC})$, the relative enhancement factor at $\mathrm{Pt}$ substrate to that at Au substrate is estimated to be one-third. The absolute enhancement factor in this system depends on the numerical aperture of the objective lens. This is because the fraction of $p$-polarization component in the (a) $\operatorname{Pt}(111)$

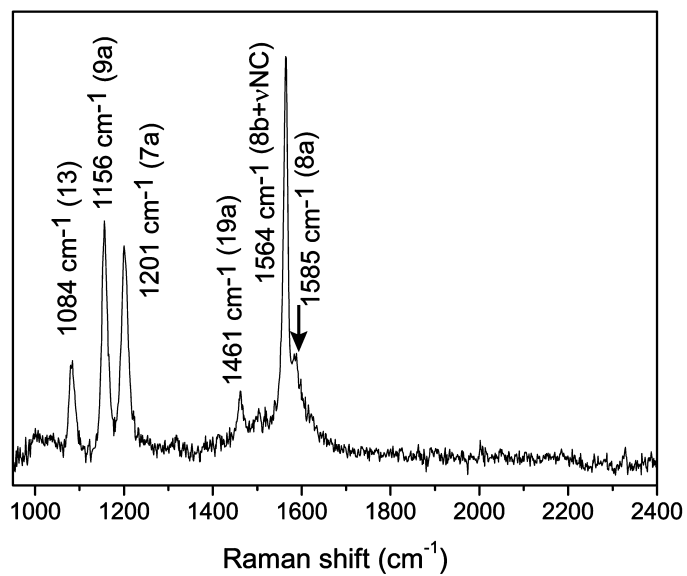

(b) $\mathrm{Au}(111)$

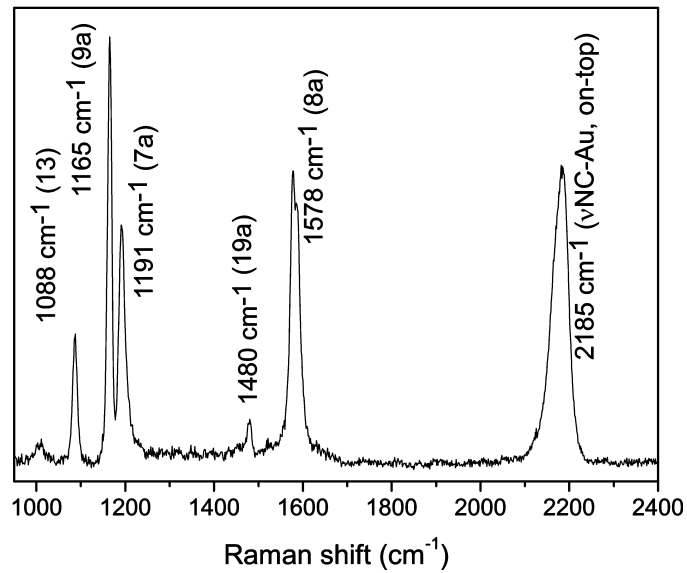

Figure 6. Gap-mode enhanced Raman spectra of CPI-SAM at (a) $\mathrm{Pt}(111)$ facet and (b) $\mathrm{Au}(111)$ facet.

excitation beam, which can couple with gap-mode plasmons, is increased with the increase of the aperture. Moreover, the molecular packing density at $\mathrm{Pt}(100)$ or $\mathrm{Au}(100)$ have not been reported. However, the enhancement factor at $\mathrm{Au}(100)$ is expected to be same as that at $\mathrm{Au}(111),{ }^{36}$ i.e., $4 \times 10^{5}$, in the present experimental setup because the electric field enhancement should not be crystal-face dependent. Since the relative enhancement factor at $\operatorname{Pt}(100)$ to that at $\mathrm{Au}(100)$ is one-third as already estimated, the absolute enhancement factor at the non-SERS-active Pt is roughly on the order of $10^{5}$. This is 3 orders of magnitude larger than the previously reported value., ${ }^{9}, 10$ Such a large effect in this technique must be due to the utilization of the localized plasmons and makes this technique a practical method for Raman spectroscopy on non-SERS-active substrates.

Since Raman signals are enhanced so significantly by the gapmode plasmon excitation that crystal-face dependence of Raman spectra, i.e., adsorbed molecular structures, can be investigated. It is again emphasized here that such an experiment is impossible in conventional SERS. Figure 6 shows Raman spectra of CPI at $\mathrm{Pt}(111)$ and $\mathrm{Au}(111)$ facets measured by the gap-mode Raman technique. The spectrum at the $\operatorname{Pt}(111)$ surface (Figure 6a) is significantly different from that at $\mathrm{Pt}(100)$ surface (Figure 5a). In the spectrum at $\mathrm{Pt}(111)$ surface, no $v \mathrm{NC}$ peak was observed in the wavenumber region between 1800 and $2200 \mathrm{~cm}^{-1}$ and a nontotally symmetric $\nu \mathrm{C}=\mathrm{C}$ peak (8b) was much larger than the totally symmetric $v \mathrm{C}=\mathrm{C}$ peak (8a). These features suggest 
(a) (i)

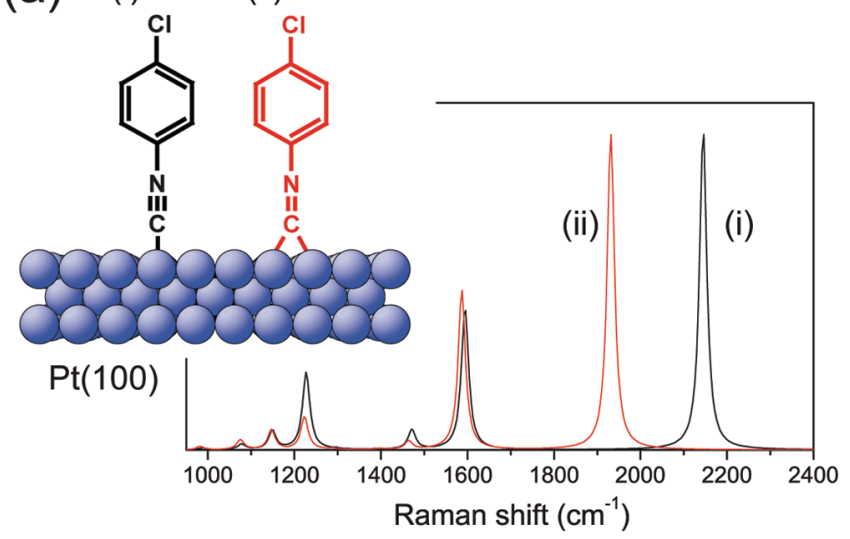

(b)

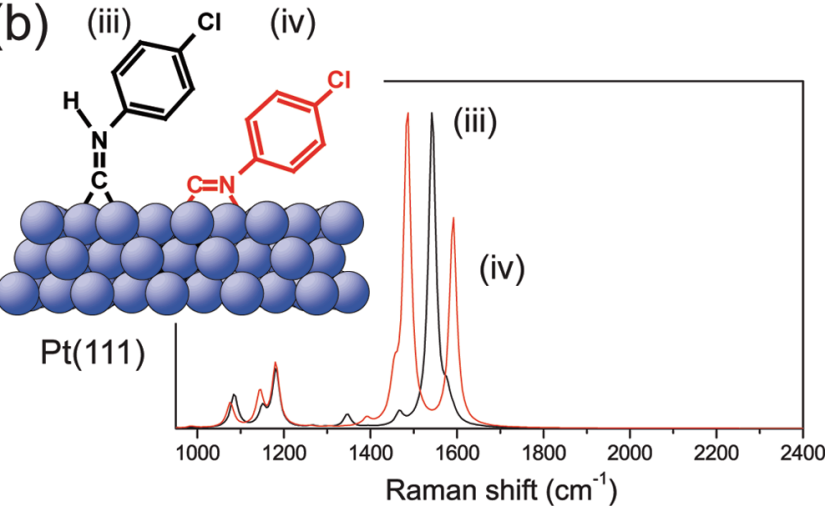

Figure 7. Calculated Raman spectra of CPI at Pt substrates, by the DFT method, for (a) on-top and bridge configurations and for (b) $\mathrm{N}$-protonated and $\eta^{2}$-bonding configurations.

that the binding of CPI molecules at $\operatorname{Pt}(111)$ surface is different from that at $\operatorname{Pt}(100)$ surface. On the other hand, Raman spectra of $\mathrm{CPI}$ at $\mathrm{Au}(111)$ surface (Figure 6b) is similar to that at the $\mathrm{Au}(100)$ surface (Figure 5b).

In order to discuss adsorption geometries of CPI molecules, density functional theory (DFT) calculations were carried out using the Gaussian 03, revision E.01, at the B3PW91 level of DFT with LanL2DZ basis set for Au and Pt atoms and 6-31G** basis sets for other atoms. The $(\mathrm{Au})_{2}$ or $(\mathrm{Pt})_{2}$ cluster was used in the calculations as a model of metal substrates. As possible adsorption models, ${ }^{37,38}$ four types of binding configurations were examined: (i) on-top, (ii) bridge, (iii) $\mathrm{N}$-protonation, and (iv) $\eta^{2}$-bonding (see Figure 7). The experimental and calculated vibrational modes are summarized in Table 1 . As for the binding configurations on $\mathrm{Au}(111)$ and (100) surfaces, the calculated result for on-top adsorption (i) agreed with the experimentally obtained result. ${ }^{16}$ Similarly, the on-top configuration (i) is dominant on the $\operatorname{Pt}(100)$ surafce. Besides, the bridge configuration (ii) is also present on this surface. On the $\mathrm{Pt}(111)$ surafce, however, these two structures are negligible, as clearly indicated by the absence of the $v \mathrm{NC}$ vibrations in the spectra (Figure 6a). The models (iii) and (iv) suggest no peak in the $v \mathrm{NC}$ vibration region (Figure 7b). The difference between the latter two models is found in the $v \mathrm{C}=\mathrm{C}$ vibration region. In the model (iii), the totally symmetric $v \mathrm{C}=\mathrm{C}$ vibration (8a) loses intensity while the nontotally symmetric $v \mathrm{C}=\mathrm{C}$ vibration (8b) gains intensity via the coupling with $v \mathrm{NC}$. On the other hand, the model (iv) additionally shows the very intense 19 a in the $v \mathrm{C}=\mathrm{C}$ vibration region with the coupling of $v \mathrm{NC}$. Thus, one can conclude that the model (iii) is more consistent with the experimental result. When CPI adsorbs on $\mathrm{Pt}(111)$ with the structure (iii), $v \mathrm{NH}$ should appear around $3420 \mathrm{~cm}^{-1}$ according to the calculation. Unfortunately, we were not able to detect this peak, maybe because the sensitivity in the present measurement system is rather low at this higher frequency region and Raman intensity of $v \mathrm{NH}$ mode is weak. However, there are some reports concerning $\mathrm{N}$-protonated adsorption forms of isocyanide molecules on $\mathrm{Pt}(111) .{ }^{39-41}$

It is known that molecular adsorption on metal surfaces is well explained by the d-band theory; the energy center of the valence d-band density of states at the surface sites correlates with their ability to form bonds with chemisorbed molecules. ${ }^{42,43}$ In the present case, $\operatorname{Pt}(111)$ has the highest d-band center position among the four crystal faces. ${ }^{44,45}$ As the d-band center shifts up, $\pi$ back-donation from metal into CPI is increased, resulting that the metal- $\mathrm{C}$ bond becomes stronger and $\mathrm{N}$ protonated form (iii) is stabilized. ${ }^{46}$

\section{Conclusion}

Significant enhancement of Raman scattering intensities of organic monolayers on non-SERS-active substrates by means of gap-mode plasmon excitation was demonstrated for the CPI monolayer on Pt surfaces. Enhancement factor comparable to that at Au surface was achieved at Pt surface. This technique should be applicable not only for Pt but also for other nonSERS-active metals by choosing appropriate excitation wavelength depending on combination of a metal substrate and metal nanoparticles.

Crystal-face dependent adsorbed structures were investigated at (111) and (100) surfaces of Pt and Au. Systematic study on molecular adsorption at step-edges and kink sites, which is not possible by conventional SERS, is under way using high-index planes.

TABLE 1: Experimental and Calculated Vibrational Frequencies $\left(\mathrm{cm}^{-1}\right)$ of CPI Adsorbed on Pt and Au Substrates ${ }^{a}$

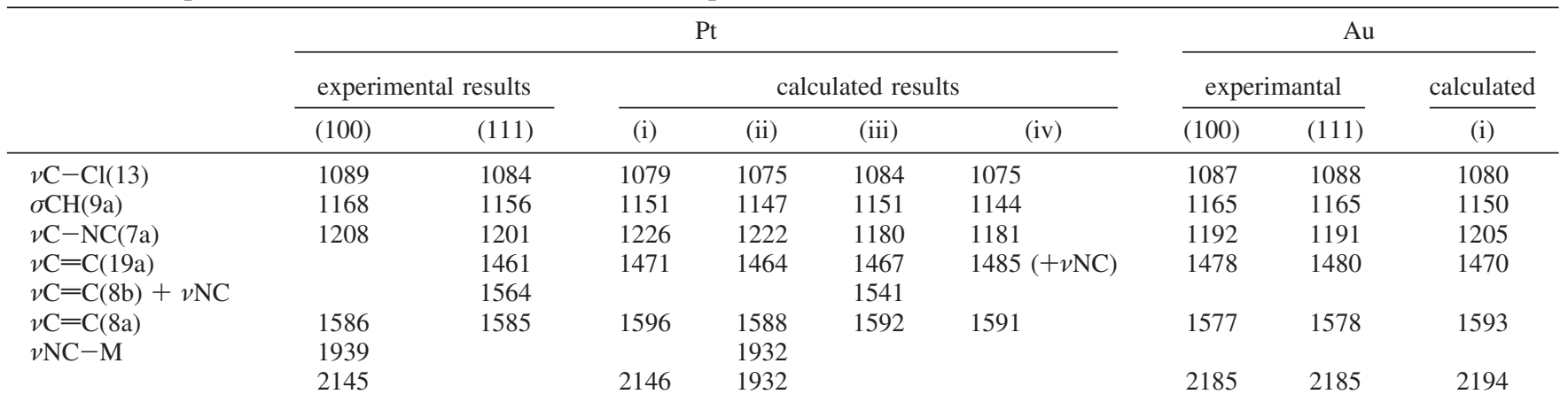

${ }^{a}$ The calculated models (i), (ii), (iii), and (iv) correspond to the on-top, bridge, N-protonation, and $\eta^{2}$-bonding configurations, respectively. 
Furthermore, since Raman spectroscopy sensitively reflects electronic information of metal-molecular interfaces as well as structural information of adsorbed molecules, ${ }^{1,2}$ the gap-mode Raman spectroscopy should be a powerful tool for detailed understanding of catalytic reaction mechanism.

Acknowledgment. This research was partially supported by Grant-in-Aid for Scientific Research (A) (2006-2009, No. 18205016) from JSPS, and that on Priority Area "Strong PhotonMolecule Coupling Fields (No. 470), World Premier International Research Center (WPI) Initiative on Materials Nanoarchitechtonics and Global COE program (Project No. B01: Catalysis as the Basis for Innovation in Materials Science) from Ministry of Education, Culture, Sports, Science and Technology (MEXT), Japan. The SEM measurement was supported by Hokkaido Innovation through NanoTechnology Support (HINTS, Nanotechnology Network Project supported by MEXT, Japan).

Supporting Information Available: SERS spectrum and AFM image $(500 \mathrm{~nm} \times 500 \mathrm{~nm})$ of CPI-SAM/Au-NPs. This material is available free of charge via the Internet at http:// pubs.acs.org.

\section{References and Notes}

(1) Otto, A.; Mrozek, I.; Grabhorn, H.; Akemann, W. J. Phys.: Condens. Matter 1992, 4, 1143-1212.

(2) Moskovits, M. Rev. Mod. Phys. 1985, 57, 783-826.

(3) Fleishmann, M.; Hendra, P. J.; McQuillan, A. J. Chem. Phys. Lett. 1974, 26, 163-166.

(4) Jeanmaire, D. L.; Van Duyne, R. P. J. Electroanal. Chem. 1977, $84,1-20$. 5217 .

(5) Albrecht, M. G.; Creighton, J. A. J. Am. Chem. Soc. 1977, 99, 5215-

(6) Kneipp, K.; Wang, Y.; Kneipp., H.; Perelman, L. T.; Itzkan, I.; Dasari, R. R.; Feld, M. S. Phys. Rev. Lett. 1997, 78, 1667-1669.

(7) Nie, S.; Emory, S. R. Science 1997, 275, 1102-1106.

(8) Seki, H. J. Electron Spectrosc. Relat. Phenom. 1986, 39, 289-310.

(9) Futamata, M.; Keim, E.; Bruckbauer, A.; Schumacher, D.; Otto, A. Appl. Surf. Sci. 1996, 100/101, 60-63.

(10) Abdelsalam, M. E.; Bartlett, P. N.; Baumberg, J. J.; Russell, A. E. J. Am. Chem. Soc. 2007, 129, 7399-7406.

(11) Weaver, M. J.; Zou, S. Z.; Chan, H. Y. H. Anal. Chem. 2000, 72, $38 \mathrm{~A}-47 \mathrm{~A}$.

(12) Neck, K. N.; Janesko, B. G.; Scuseria, G. E.; Halas, N. J.; Wong, M. S. J. Am. Chem. Soc. 2008, 130, 16592-16600.

(13) Naohara, H.; Ye, S.; Uosaki, K. J. Electroanal. Chem. 2001, 500, 435-445.

(14) Awatani, T.; Yagi, I.; Noguchi, H.; Uosaki, K. J. Electroanal. Chem. 2002, 524/525, 184-193.

(15) Kibler, L. A.; El-Aziz1, A. M.; Kolb, D. M. J. Mol. Catal. A 2003 199, 57-63.

(16) Ikeda, K.; Fujimoto, N.; Uehara, H.; Uosaki, K. Chem. Phys. Lett. 2008, 460, 205-208.

(17) Aravind, P. K.; Metiu, H. J. Phys. Chem. 1982, 86, 5076-5084.

(18) Aravind, P. K.; Metiu, H. Sur. Science 1983, 124, 506-528.

(19) Driskell, J. D.; Lipert, R. J.; Porter, M. D. J. Phys. Chem. B 2006, 110, 17444-17451.

(20) Pettinger, B.; Ren, B.; Picardi, G.; Schuster, R.; Ertl, G. Phys. Rev. Lett. 2004, 92, 096101.
(21) Hayazawa, N.; Ishitobi, H.; Taguchi, A.; Tarun, A.; Ikeda, K.; Kawata, S. Jpn. J. Appl. Phys. 2007, 46, 7995-7999.

(22) Wind, M. M.; Vlieger, J. Physica A 1987, 141, 33-57.

(23) Prodan, E.; Radloff, C.; Halas, N. J.; Nordlander, P. Science 2003, $302,419-422$.

(24) Nordlander, P.; Oubre, C.; Prodan, E.; Li, K.; Stockman, M. I. Nano Lett. 2004, 4, 899-903.

(25) Okamoto, T.; Yamaguchi, I. J. Phys. Chem. B 2003, 107, 1032110324.

(26) The validity of the continuum dielectric approximation may be broken when the cell size in the FDTD calculation is too small. Therefore, the gap distance of $1.5 \mathrm{~nm}$ and the Au-sphere diameter of $50 \mathrm{~nm}$ were chosen for the demonstration.

(27) Pettinger, B. J. Chem. Phys. 1986, 85, 7442-7451.

(28) Clavilier, J.; Faure, R.; Guinet, G.; Durand, R. J. Electroanal. Chem. 1980, 107, 205-209.

(29) Komanicky, V.; Chang, K. C.; Menzel, A.; Markovic, N. M.; You, H.; Wang, X.; Myers, D. J. Electrochem. Soc. 2006, 153, B446-B451.

(30) Frens, G. Nat. Phys. Sci. 1973, 241, 20.

(31) Lee, J.-O.; Lientschnig, G.; Wiertz, F.; Struijk, M.; Janssen, R. A. J.; Egberink, R.; Reinhoudt, D. N.; Hadley, P.; Dekker, C. Nano Lett. 2003, 3, 113-117.

(32) Hong, S.; Reifenberger, R.; Tian, W.; Datta, S.; Henderson, J.; Kubiak, C. P. Superlattices Microstruct. 2000, 28, 289-303.

(33) Gruenbaum, S. M.; Henney, M. H.; Kumar, S.; Zou, S. J. Phys. Chem. B 2006, 110, 4782-4792.

(34) Raman spectrum of CPI monolayer formed on the surface of $\mathrm{Au}$ nanoparticles that are deposited on a glass substrate is presented as Supporting Information together with AFM image $(500 \mathrm{~nm} \times 500 \mathrm{~nm})$ of CPI-SAM/Au NPs deposited on the glass substrate. Since the CN anchor groups are bound to Au in this case, the frequency of $v \mathrm{NC}$ is same as that in the Au-sphere/CPI/Au-plane. Moreover, Raman intensity per nanoparticle is 20 times stronger in the Au-sphere/CPI/Au-plane than in CPI/Au NPs on glass. Thus, it is confirmed that the Raman signals in the Au-sphere/ CPI/Pt-plane are enormously enhanced at the gap regions.

(35) The $\nu \mathrm{C}=\mathrm{C}$ peak at the $\mathrm{Au}(100)$ is broader than the corresponding peak at the $\mathrm{Pt}(100)$ surface. Since this vibrational frequency is affected by $\pi-\pi$ interactions between aromatic rings, the observed difference implies that two-dimentional packing arrangement is different on these metal substrates. However, such a difference does not affect the estimation of the enhancement factor as long as both the packing density and the adsorption geometry are determined. Details of the two-dimentional arrangement may be studied by STM.

(36) The number of molecules showing the enhanced Raman signal at Au(111) is estimated to be 250 molecules per hot spot based on the molecular packing density measured by the quartz microbalance method (Kim, H. S.; Lee, S. J.; Kim, N. H.; Yoon, J. K.; Park, H. K.; Kim, K. Langmuir 2003, 19, 67016710) .

(37) Avery, N. R.; Matheson, T. W. Surf. Sci. 1984, 143, 110-124.

(38) Murphy, K. L.; Tysoe, W. T.; Bennett, D. W. Langmuir 2004, 20, $1732-1738$.

(39) Kang, D.-H.; Trenary, M. J. Am. Chem. Soc. 2001, 123, 84328433.

(40) Mudiyanselage, K.; Trenary, M.; Meyer, R. J. J. Phys. Chem. 2008, $112,3794-3799$.

(41) Katano, S.; Kim, S.; Hori, M.; Trenary, M.; Kawai, M. Science 2007, 316, 1883-1886.

(42) Hammer, B.; Nørskov, J. K. Surf. Sci. 1995, 343, 211-220.

(43) Greeley, J.; Nørskov, J. K.; Mavrikakis, M. Annu. Rev. Phys. Chem. 2002, 53, 319-348.

(44) Stamenkovic, V. R.; Fowler, B.; Mun, B. S.; Wang, G.; Ross, P. N.; Lucas, C. A.; Marković, N. M. Science 2007, 315, 493-497.

(45) Roudgar, A.; Gross, A. J. Electroanal. Chem. 2003, 548, 121130.

(46) Michelin, R. A.; Pombeiro, A. J. L.; Guedes da Silva, M. F. C. Coord. Chem. Rev. 2001, 218, 75-112.

JP901858T 